\title{
Influence of several sources of cocoa flavonoids on humoral immune system in young rats
}

\author{
M. Massot-Cladera, M. Abril-Gil, T. Pérez-Berezo, K. Becerra, S. Torres, A. Franch, F. J. Pérez-Cano \\ and M. Castell \\ Department of Physiology, Faculty of Pharmacy, University of Barcelona; Institut de Recerca en Nutrició $i$ Seguretat \\ Alimentària (INSA-UB), Barcelona, Spain
}

Experimental evidence suggests that cocoa polyphenols have immunomodulatory potential; however, most of the studies evaluating these effects are performed in vitro. In previous studies we have reported that a $10 \%$ cocoa diet containing about $0.2 \%$ (w/w) of polyphenols produced an attenuating effect on the synthesis of mucosal and systemic immunoglobulins) in young rats ${ }^{(1-3)}$. This modulatory effect could be attributed to flavonoids or other compounds present in cocoa such as the fibre or methylxanthines. The aim of the present study was to ascertain whether different sources of cocoa, which have higher amounts of flavonoids but lacking fibre or met hylxanthines, were able to reproduce this effect on intestinal $\operatorname{IgA}$ and serum $\operatorname{IgG}$ antibodies in two strains of rats.

Weaned female Brown Norway and Wistar rats were fed either standard diet, isoenergetic diet containing $0.2 \%$ cocoa polyphenols (mainly procyanidins) from conventional defatted cocoa (Nutrexpa SL) or isoenergetic diet containing $0.4 \%$ or $0.8 \%$ cocoa polyphenols (mainly monomeric flavanols) from non-fermented cocoa (Natraceutical Group), during four weeks. Faecal and serum samples were obtained along the study and total $\operatorname{IgA}$, IgG1, IgG2a and $\operatorname{IgG} 2 \mathrm{~b}$ were quantified by ELISA.

Results show that all polyphenol-enriched cocoa diets produced attenuation on intestinal IgA in both strains although in a doseindependent manner. However, in serum IgG, Wistar rats were more sensitive to conventional cocoa than non-fermented cocoa. In conclusion, cocoa diets influence immunoglobulin production but these effects do not seem to be exclusive of polyphenols but also dependent on the other cocoa compounds present in cocoa.

1. Ramiro-Puig E, Pérez-Cano FJ, Ramírez-Santana C, et al. (2007) Clin Exp Immunol 149, 535-542.

2. Ramiro-Puig E, Pérez-Cano FJ, Ramos-Romero S, et al. (2008) J Nut Biochem 19, 555-565.

3. Pérez-Berezo T, Franch A, Ramos-Romero S, et al. (2011) Mol Nutr Food Res 55S, 56-66. 